\title{
Anti-CTLA-4 Induced Inflammatory Bowel Disease: Is There A More Etiological Treatment? Lessons From CTLA-4 Haploinsufficiency
}

\begin{abstract}
Athina Theocharidou and Georgios Germanidis*
First Department of Internal Medicine, Gastroenterology and Hepatology Section, AHEPA Hospital of the Aristotle University Medical School, Thessaloniki, Greece

*Corresponding author: Georgios Germanidis, First Department of Internal Medicine, Gastroenterology and Hepatology Section,AHEPA Hospital of the Aristotle University Medical School,1, St. Kyriakidi Str., 54636 Thessaloniki, Greece
\end{abstract}

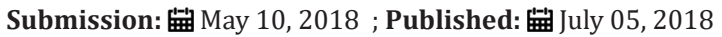

\section{Opinion}

We read with great interest the article by Bamias $\mathrm{G}$ et al. [1] entitled "Immunological Characteristics of Colitis Associated with Anti-CTLA-4 Antibody Therapy" [1], and we would like to address some issues regarding possible future use of a more etiological treatment for this colitis, namely abatacept. The immunological characteristics of anti-CTLA-4 and anti-PD-1-related colitis have been up to now poorly described $[1,2]$.

Cytotoxic T lymphocyte antigen-4 (CTLA-4) is an inhibitory T cell receptor, similar to CD28 in structure, expressed by activated and regulatory $\mathrm{T}$ cells (Tregs). CTLA-4 is constitutively expressed on CD4+CD25+ Tregs, and such expression is important for Tregmediated suppression of $\mathrm{T}$ cell proliferation. Abatacept is a soluble fusion protein which links the CTLA-4 extracellular domain to the Fc region of the IgG molecule. CTLA-4 immunoglobulin (Ig) fusion protein and neutralizing CTLA-4 antibody are used to modulate immunity in autoimmune and cancer patients, respectively.

Recently in two different reports, germline heterozygous mutations of CTLA- 4 in subjects with severe immune dysregulation from unrelated families were identified [3,4]. Human CTLA-4 haploinsufficiency caused dysregulation of FoxP3+regulatory $\mathrm{T}$ (Treg) cells, hyperactivation of effector $\mathrm{T}$ cells, severe immunoregulatory disorder, and autoimmune lymphocytic infiltration of target organs. Patients also exhibited progressive loss of circulating B cells, associated with an increase of predominantly auto-reactive CD21lo B cells and accumulation of B cells in nonlymphoid organs.

Although the frequency of FoxP3+ Treg cells within the CD4+ T cell compartment was increased in individuals with a heterozygous CTLA4 mutation compared to healthy CTLA4+/+ controls, affected patients had reduced CTLA-4 protein (similarly as to use a neutralizing CTLA-4 antibody) and mRNA expression in sorted Treg cells relative to healthy donors; this reduction persisted, and deficit was even more pronounced after $\mathrm{T}$ cell activation [3]. These results demonstrate the complex spectrum of clinical complications that can be anticipated from CTLA-4-blocking drugs. Consistent with these findings, treatment with the CTLA-4 mimetic, CTLA-4-Ig (abatacept), suppressed patients $\mathrm{T}$ cell hyperproliferation in vitro and could be a potential therapeutic intervention for CTLA-4deficient patients [3]. Therefore, human CTLA-4 haploinsufficiency demonstrates a critical quantitative role for CTLA- 4 in governing $\mathrm{T}$ and B lymphocyte homeostasis [3]. CTLA-4 can function by removal of its ligands (CD80 and CD86) from antigen presenting cells via transendocytosis.

These CTLA-4 ligands are shared with the stimulatory receptor CD28, whose engagement drives $\mathrm{T}$ cell activation, cytokine production and memory T cell differentiation. Depletion of the costimulatory ligands CD80 and CD86 by CTLA- 4 reduces antigen presenting cell-mediated activation of conventional $\mathrm{T}$ cells via CD28, resulting in dominant suppression of $\mathrm{T}$ cell activation [4]. Treg cells from individuals with CTLA4 heterozygous mutations were unable to suppress $\mathrm{CD} 4+\mathrm{T}$ cell proliferation as compared to healthy CTLA4+/+ controls.

Taken together, these data indicate that two functional CTLA4 alleles appear necessary to drive the high levels of protein required in activated Treg cells, and in this condition their overall suppressive activity is impaired by CTLA-4 mutations. The relative role of CTLA4 in Treg cells versus conventional $\mathrm{T}$ cells remains unexplained and additional work is needed to interprete the $\mathrm{T}$ cell-intrinsic hyperproliferation in these patients bearing CTLA4 mutations. It is unclear whether the hyperproliferative $\mathrm{T}$ cell phenotype reported is due to loss of CTLA-4 function in conventional T cells. Clinical autoimmune features phenotype of organs with inflammatory infiltrates, include mostly the brain, lung, gut and cytopenias. In most patients, biopsies of the gastrointestinal tract revealed histopathology similar to that caused by CTLA-4 blocking antibody treatment in melanoma patients [5-7]. 
Bamias $G$ et al. [1] by investigating for the first time the mucosa immunophenotype of colitis associated with anti-CTLA-4 antibody therapy, showed that ipilimumab-associated colitis is characterized by expansion of infiltrating CD4+ lymphocytes and plasma cells, along with mucosal predominance of Th1 and Th17 effector cytokines. Significant elevation of FoxP3 was also detected, although with possible cellular defects on the functional level, compromising the ability of Tregs to control effector immunity, an event well described in cases of enterocolitis in CTLA-4 haploinsufficiency $[3,4]$.

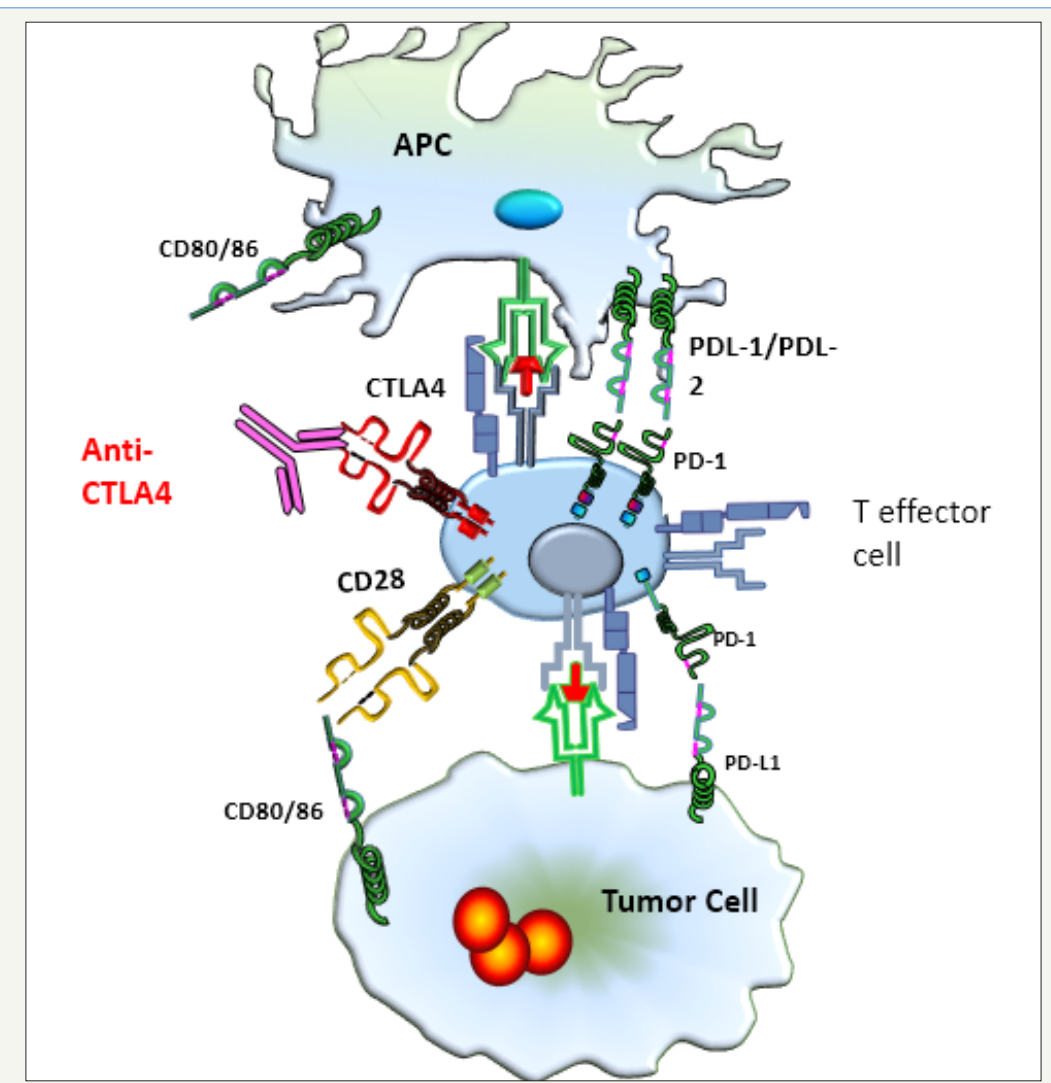

Figure 1A: Tumor immunotherapy. Anti-CTLA-4 by inhibiting the interaction of the inhibitory signal 2, CTLA-4 with CD80/ CD86, drives the balance to the augmentation of the positive signal 2, the CD28 with CD80/CD86 interaction between antigen presenting cells (APCs) and T effector cells, increasing the T cell effector function against tumor cells.

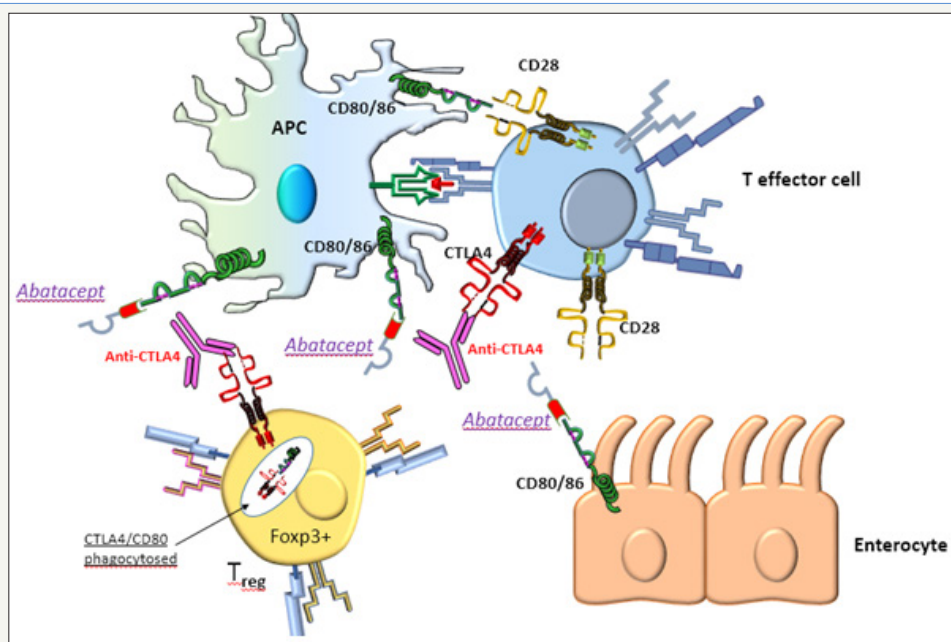

Figure 1B: Anti-CTLA-4 induced colitis and possible use of abatacept. Anti-CTLA-4 by inhibiting the interaction of CTLA-4 with CD80/CD86 between Foxp3+ve Tregs and APCs, leads to loss of regulatory function on T effector cells and hyperactivation of effector T cells against enterocytes, similarly to a heterozygous CTLA4 mutation (CTLA-4 haploinsufficiency). The relative role of CTLA-4 in Treg cells versus conventional T cells remains unexplained. Abatacept (CTLA4 -Ig) is a CTLA-4 agonist, that restores $\mathrm{T}$ cell hyperactivation and alleviates autoimmune disease in germline or de novo CTLA-4 haploinsufficiency $[3,4,12]$. Abatacept could restore T regulatory suppressor function impaired in anti-CTLA-4 induced colitis, acting more on Tregs than conventional $\mathrm{T}$ cells. However, abatacept, if used for severe anti-CTLA-4 induced colitis or enteritis, could negate the antitumor effects of anti-CTLA-4 therapy. Figure courtesy by Prof. P. Vlachoyiannopoulos. 
In addition, in both IBD and ipilimumab colitis, Bamias G et al. [1] observed high upregulation of TL1A, a TNF like cytokine that is expressed on antigen-presenting cells under the influence of microbial-induced signals. Immunotherapy associated colitis is endoscopically and histologically strikingly similar to IBD. The treatment of patients with ipilimumab-associated diarrhea according to the international guidelines today, is corticosteroid administration followed by anti-TNF- $\alpha$ agents in corticosteroidrefractory cases (Figure 1A \& 1B).

There are three points that should be examined:

a. Is there a connection between CTLA- 4 and TNF- $\alpha$ ?

In rheumatoid arthritis there is plenty of evidence that CTLA-Ig (abatacept) acts directly on macrophages through possibly the B7 receptors of macrophages (CD80/CD86 or B7-H3) with resultant downregulation of TNF- $\alpha$ production, along with IL-1 $\beta$ and IL-6 down regulation [8,9]. CTLA-4 Ig decreases NF- $\mathrm{B}$ in macrophages [10]. Although direct evidence in IBD macrophages do not exist, it is logical to assume that CTLA-Ig cause the same effects. Therefore, inhibition of CTLA-4 in all probability leads to the opposite effect i.e increase in TNF- $\alpha$ production. This might explain the favorable effect of anti- TNF- $\alpha$ in this form of colitis but more importantly raises the possibility of using abatacept for treatment of both anti-CTLA-4 colitis and IBD. Furthermore, high mucosal TNF- $\alpha$ concentrations were observed in anti-CTLA-4 -induced colitis and low mucosal TNF $\alpha$ concentrations were associated with steroid sensitivity [6].

b. What is the underlying genetical background of cancer patients that develop enterocolitis under CTLA-4 inhibition?

Although a recent meta-analysis suggests that the CTLA$4+49 \mathrm{~A} / \mathrm{G}$ exon 1 variant may be related to CD susceptibility in Caucasians, and the CTLA-4 CT60 variant may be associated with UC susceptibility in Asians [11], none of these mutations have relation with human CTLA-4 haploinsufficiency or the binding site of anti-CTLA-4 agents. It is true that there is a high prevalence of CTLA-4 associated colitis, but this varies extensively among studies [7]. Therefore, it should be interesting to examine the genetic background of the immunotherapy associated patients, since it may well be that ipilimumab was only a trigger of IBD in a genetically susceptible individual.

c. Which would be the best approach of confirmation for immunotherapy -induced colitis response to treatment in general, or with CTLA-Ig (abatacept)?

The evaluation of the complete response to any treatment with steroids, anti-TNF-a agents, or, as we propose with CTLA - Ig, today, remains really narrow. Based on the current data, we suggest that the consideration either of flex-sigmoidoscopy or colonoscopy, followed by spontaneous multisite biopsies after an established clinical benefit/response to treatment should be examined. With that clinical practice, in theory, we could investigate the level of infiltrating CD4+ lymphocytes and plasma cells, differentially, along with mucosal predominance of Th1 and Th17 effector or other cytokines and molecules, as discussed above, as well as, the differential expression of FoxP3 and other factors. Though we do believe that neither of the above can be characterized as 'biomarker' for this specific clinical condition, till today, this approach may reveal the immunopathogenesis of this entity and key molecules that can be used as markers of drug effects and response in the future.

In a recent report [12], administration of abatacept in a patient with autoimmune enteropathy carrying a de novo variant in CTLA-4 down-regulated immune responses, eventually achieving substantial clinical improvement. Previous administration of several immunosuppressants failed to achieve substantial improvement. Abatacept increased TGF- $\beta 1$ expression level, demonstrating that the drug successfully restored Treg-cell functionality. The drug treatment also restored CD3+ T-cell level and attenuated inflammatory cytokine production such as IL-10, IL-17A, and IL-6 from patient-derived PBMCs. Initial serum levels of IL-10, IL-17A, and IL- 6 were higher in the patient than in her healthy parents, and the increased serum cytokine levels were reduced in a similar fashion by the drug treatment.

In view of the above immunological and clinical data it is reasonable to assume that the use of the soluble CTLA-4-Ig agent abatacept is a potential more etiological therapeutic solution in difficult cases of anti-CTLA-4 induced inflammatory bowel disease. However, we have to notice that despite the striking similarity of ipilimubab- associated colitis with IBD as described by Bamias et al. [1] the studies showed that abatacept is not efficacious for the treatment of moderate-to-severe Crohn's disease or ulcerative colitis [13]. Abatacept, if used for severe anti-CTLA-4 induced colitis or enteritis, could negate the antitumor effects of anti-CTLA-4 therapy. In this case, swich to another agent, i.e anti-PD-1, can be an effective therapeutic strategy, since anti-CTLA-4 and anti-PD-1 blockade induce distinct immunopathological entities of colitis [2].

Recently, it has been shown in transgenic mice that c-MAFdependent, induced regulatory $\mathrm{T}$ cells (iTregs), mediate immunological tolerance in large intestine to a gut pathobiont. These ROR $\boldsymbol{\gamma t}+\mathrm{FOXP3}+$ iTreg cells selectively restrain proinflammatory, colitogenic T helper 17 (TH17) cells and their function is dependent on the transcription factor c-MAF [14]. In this important iTreg-TH17 homeostasis, c-MAF integrates antiinflammatory TGF $\beta$ receptor signals with microorganism induced cytokine-dependent STAT3 activation to mediate $\mathrm{ROR} \gamma \mathrm{t}+$ Treg induction. The role of the CTLA-4 molecule perturbation in this specific, fully functional subset of iTregs, is at present unknown, but potentially very important.

\section{References}

1. Bamias G, Delladetsima I, Perdiki M, Siakavellas SI, Goukos D, et al. (2017) Immunological characteristics of colitis associated with antiCTLA-4 antibody therapy. Cancer Invest 35(7): 443-455.

2. Coutzac C, Adam J, Soularue E, Collins M, Racine A, et al. (2017) Colon immune-related adverse events: anti-CTLA-4 and anti-PD-1 blockade induce distinct immunopathological entities. J Crohns Colitis 11(10): 1238-1246.

3. Kuehn HS, Ouyang W, Lo B, Deenick EK, Niemela JE, et al. (2014) Immune dysregulation in human subjects with heterozygous germline mutations 
in CTLA4. Science 345(6204): 1623-1627.

4. Schubert D, Bode C, Kenefeck R, Hou TZ, Wing JB, et al. (2014) Autosomal dominant immune dysregulation syndrome in humans with CTLA4 mutations. Nat Med 20(12): 1410-1416.

5. Larkin J, Chiarion-Sileni V, Gonzalez R, Grob JJ, Cowey CL, et al. (2015) Combined nivolumab and ipilimumab or monotherapy in untreated melanoma. N Engl J Med 373(1): 23-34.

6. Marthey L, Mateus C, Mussini C, Nachury M, Nancey S, et al. (2016) Cancer immunotherapy with anti-CTLA-4 monoclonal antibodies induces an inflammatory bowel disease. J Crohns Colitis 10(4): 395-401.

7. Gupta A, De Felice KM, Loftus EV, Khanna S (2015) Systematic review: colitis associated with anti-CTLA-4 therapy. Aliment Pharmacol Ther 42(4): 406-417.

8. Cutolo M, Soldano S, Montagna P, Sulli A, Seriolo B, et al. (2009) CTLA4-Ig interacts with cultured synovial macrophages from rheumatoid arthritis patients and downregulates cytokine production. Arthritis Res Ther 11(6): 176

9. Wenink MH, Santegoets KC, Platt AM, van den Berg WB, van Riel PL, et al. (2012) Abatacept modulates proinflammatory macrophage responses upon cytokine-activated T cell and Toll-like receptor ligand stimulation. Ann Rheum Dis 71(1): 80-83.

10. Castellanos JR, Purvis IJ, Labak CM, Guda MR, Tsung AJ, et al. (2017) B7$\mathrm{H} 3$ role in the immune landscape of cancer. Am J Clin Exp Immunol 6(4): 66-75.

11. Zhang M, Ni J, Xu WD, Wen PF, Qiu LJ, et al. (2014) Association of CTLA4 variants with susceptibility to inflammatory bowel disease: a metaanalysis. Hum Immunol 75(3): 227-233.

12. Lee S, Moon JS, Lee CR, Kim HE, Baek SM, et al. (2016) Abatacept alleviates severe autoimmune symptoms in a patient carrying a de novo variant in CTLA-4. J Allergy Clin Immunol 137(1): 327-330.

13. Sandborn WJ, Colombel JF, Sands BE, Rutgeerts P, Targan SR, et al. (2012) Abatacept for Crohn's disease and ulcerative colitis. Gastroenterology 143(1): 62-69.

14.Xu M, Pokrovskii M, Ding Y, Yi R, Au C, et al. (2018) c-Maf-dependent regulatory $\mathrm{T}$ cells mediate immunological tolerance to a gut pathobiont. Nature 554(7692): 373-377.
Creative Commons Attribution 4.0

International License

For possible submissions Click Here

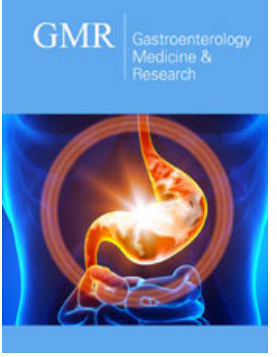

\section{Gastroenterology Medicine \& Research}

\section{Benefits of Publishing with us}

- High-level peer review and editorial services

- Freely accessible online immediately upon publication

- Authors retain the copyright to their work

- Licensing it under a Creative Commons license

- Visibility through different online platforms 